\title{
GENERALIZED HODOGRAPH TRANSFORMATION AND ITS APPLICATION TO FREE BOUNDARY PROBLEMS
}

\author{
SHUXING CHEN* AND DENING $\mathrm{LI}^{\dagger}$
}

1. Generalized Hodograph Transformation. In the study of free boundary problems for partial differential equations the moving boundary often causes much difficulty. One way to deal with this difficulty is to introduce a transformation to fix the boundary. In this paper we would like to introduce a generalized hodograph transformation which has been successively applied to treat the problems of supersonic flow passing a pointed body.

Hodograph transformation was first introduced in the study of gas dynamics (for instance, see [2]). For steady two-dimensional irrotational flow, the governing system of differential equations is

$$
\left\{\begin{array}{l}
v_{x}-u_{y}=0 \\
(\rho u)_{x}+(\rho v)_{y}=0
\end{array}\right.
$$

where $(u, v)$ is the velocity in the flow field, and $\rho$ is the density. The second equation can also be written as

$$
\left(c^{2}-u^{2}\right) u_{x}-u v\left(u_{y}+v_{x}\right)+\left(c^{2}-v^{2}\right) v_{y}=0
$$

where $c$ stands for the sonic speed. (1.1) is a quasilinear differential system. Many problems in gas dynamics demand its solution, for instance, the determination of the flow field around a given wing in aerodynamics. It is often difficult to solve directly boundary value problems of such a nonlinear differential system. Fortunately, this system is reducible to a pair of linear differential equations by introducing $x$ and $y$ as functions of the velocity components $u$ and $v$, provided that the Jacobian

$$
j=u_{x} v_{y}-v_{x} u_{y}
$$

does not vanish. By means of the relations $u_{x}=j y_{v}, \quad u_{y}=-j x_{v}$ and $v_{x}=$ $-j y_{u}, \quad v_{y}=j x_{u}$, system (1.1) is transformed into a linear differential system

$$
\left\{\begin{array}{l}
x_{v}-y_{u}=0 \\
\left(c^{2}-u^{2}\right) y_{v}+u v\left(x_{v}+y_{u}\right)+\left(c^{2}-v^{2}\right) x_{u}=0
\end{array}\right.
$$

Generally, solving the boundary value problem of (1.3) is easier than solving the corresponding boundary value problem for the nonlinear system (1.1).

In fact, this idea of interchanging the variables and unknown functions was often employed in the theory of ordinary differential equations. Let's look at, for instance, the second order equation

$$
\frac{d^{2} y}{d x^{2}}=f\left(x, y, \frac{d y}{d x}\right)
$$

*Institute of Mathematics of Fudan University and Center of Mathematical Sciences at Zhejiang University, China.

$\dagger$ Department of Mathematics, West Virginia University, U.S.A. 
Regarding $y$ as the independent variable and $x(y)$ as the unknown function, one can reduce the equation into

$$
\frac{d^{2} x}{d y^{2}}=-\left(\frac{d x}{d y}\right)^{3} f\left(x, y,\left(\frac{d x}{d y}\right)^{-1}\right)
$$

Obviously, once a solution $x=x(y)$ of (1.5) is obtained, then its inverse $y=y(x)$ is the solution of (1.4), provided $x^{\prime}(y) \neq 0$.

This kind of transformation, which we will denote as $T$, can also be used to change a free boundary problem into a problem with fixed boundary. For instance, consider the boundary value problem of (1.4) with conditions

$$
\begin{gathered}
y=y_{1}, \quad \text { on } \quad x=x_{1} \\
y=y_{2}, g\left(x, y, \frac{d y}{d x}\right)=0, \quad \text { on } x=\alpha
\end{gathered}
$$

where $\alpha$ is to be determined. Under the transformation $T$ the boundary conditions are reduced to

$$
\begin{gathered}
x=x_{1}, \quad \text { on } \quad y=y_{1} \\
g\left(x, y,\left(\frac{d x}{d y}\right)^{-1}\right)=0, \quad \text { on } \quad y=y_{2}
\end{gathered}
$$

The problem (1.5) with (1.8),(1.9) is a normal boundary value problem of second order ordinary differential equation for function $x(y)$ with fixed boundary, hence it can be treated easily.

For partial differential equations, we can also interchange one variable with the unknown function while keeping all other variables unchanged. For instance, suppose a differential equation of the unknown function $\phi$ be defined in the domain $\phi\left(x_{1}, \cdots, x_{n}\right) \geq 0$. If we can show that $\frac{\partial \phi}{\partial x_{n}}>0$, then by introducing the transformation

$$
\begin{aligned}
& y_{i}=x_{i} \quad i=1, \cdots, n-1 \\
& y_{n}=\phi\left(x_{1}, \cdots, x_{n}\right)
\end{aligned}
$$

we are able to fix the boundary. Meanwhile, the inverse transform $x_{n}=u\left(y_{1}, \cdots, y_{n}\right)$ of the function $y_{n}=\phi\left(x_{1}, \cdots, x_{n}\right)$ will be regarded as the unknown function.

Such a transformation as in (1.10) is called partial hodograph transformation. A nice property of this transformation is that it does not change the type of the equation. Recently, partial hodograph transformation has been successfully applied to prove the existence of solution to Cauchy problem of potential gas flow equation with discontinuous initial data (see $[11,13]$ ).

In case when the condition on $x=x_{1}$ takes the general non-Dirichlet form

$$
h\left(x, y, \frac{d y}{d x}\right)=0
$$

difficulty may arise by applying the transformation (1.10). In fact, if we perform the transformation (1.10), the previously fixed boundary $x=x_{1}$ will become a new free boundary in new coordinates. To avoid the appearance of any new free boundary, we 
can use the method of domain decomposition. That is, decompose the interval $\left(x_{1}, \alpha\right)$ into overlapping intervals, then solve sub boundary value problems alternatively like people does in Schwartz alternating method. The method is available if the function $y(x)$ is a monotonically increasing function. If this is true, we can add $x_{a}, x_{b}$ in the interval $\left(x_{1}, \alpha\right)$, such that $x_{1}<x_{a}<x_{b}<\alpha$, and correspondingly, $y_{1}<y_{a}<y_{b}<y_{2}$. Then we introduce two related problems defined on $\left(x_{1}, x_{b}\right)$ and $\left(y_{a}, y_{2}\right)$ respectively. That is:

$$
\begin{aligned}
& (I):\left\{\begin{array}{l}
\frac{d^{2} y}{d x^{2}}=f\left(x, y, \frac{d y}{d x}\right) \\
y=y_{1} \text { on } x=x_{1} \\
\text { Dirichlet condition on } x=x_{b}
\end{array}\right. \\
& (I I):\left\{\begin{array}{l}
\frac{d^{2} x}{d y^{2}}=-\left(\frac{d x}{d y}\right)^{3} f\left(x, y,\left(\frac{d x}{d y}\right)^{-1}\right) \\
h\left(x, y,\left(\frac{d x}{d y}\right)^{-1}\right)=0 \text { on } y=y_{2} \\
\text { Dirichlet condition on } y=y_{a}
\end{array}\right.
\end{aligned}
$$

Both problems (I) and (II) are given on an interval with fixed boundary. Starting from an appropriate value $y^{(0)}$ on $x=x_{b}$, we can solve the problems (I) and (II) alternatively and construct two sequences of solutions by the following procedure

$$
\begin{aligned}
& \left\{\begin{array}{l}
\frac{d^{2} y^{(n)}}{d x^{2}}=f\left(x, y^{(n)}, \frac{d y^{(n)}}{d x}\right), \\
y^{(n)}=y_{1} \quad \text { on } \quad x=x_{1}, \\
y^{(n)}=\left(\operatorname{Inv}\left(x^{(n-1)}\right)\left(x_{b}\right) \text { on } \quad x=x_{b} ;\right.
\end{array}\right. \\
& \left\{\begin{array}{l}
\frac{d^{2} x^{(n)}}{d y^{2}}=-\left(\frac{d x^{(n)}}{d y}\right)^{3} f\left(x^{(n)}, y,\left(\frac{d y^{(n)}}{d x}\right)^{-1}\right), \\
h\left(x^{(n)}, y,\left(\frac{d x^{(n)}}{d y}\right)^{-1}\right)=0 \text { on } y=y_{a}, \\
x^{(n)}=\left(\operatorname{Inv}\left(y^{(n)}\right)\left(y_{a}\right) \text { on } y=y_{a} .\right.
\end{array}\right.
\end{aligned}
$$

If these sequences are convergent, the limits of (I) and (II) in the interval $\left(x_{a}, x_{b}\right)$ ( or $\left.\left(y_{a}, y_{b}\right)\right)$ will be inverse functions of each other. Then the problem (1.4),(1.9), (1.11) is solved.

As we mentioned earlier that the above method can be employed only for the case when the expected solution is monotonically increasing or decreasing. However, in some cases such a condition may not be satisfied and this leads to another approach to apply the partial hodograph transformation as follows.

In this new approach, we introduce the new variable and new unknown function in partial hodograph transformation by a combination of the original variable $x$ and the unknown function $y$. For instance, in the problem (1.4),(1.9),(1.11), $\theta=\arctan (y-$ $\left.y_{2}\right) /\left(x-x_{1}\right)$ can be chosen as new variable, while $r=\sqrt{\left(x-x_{1}\right)^{2}+\left(y-y_{2}\right)^{2}}$ can be 
chosen as new unknown function. In this case the equation (1.4) will be transformed into a second order equation for function $r(\theta)$. A different choice is to introduce

$$
z=\frac{x-x_{1}}{x-x_{1}+y-y_{2}}, \quad w=x-x_{1}+y-y_{2}
$$

where $z$ is regarded as a new variable, and $w$ is regarded as the new unknown function. Obviously, the transformation will change the boundary $x=x_{1}$ and $y=y_{1}$ to $z=$ 0 and $z=1$ respectively. In the meantime, the troublesome computation caused by square roots can be avoided. Such a transformation is also called generalized hodograph transformation.

Generalized hodograph transformation has been proved as an effective method in the discussion of the existence of solution to supersonic flow past a pointed body. In such problems the possible shock front is a free boundary which will be transformed into a fixed boundary by the generalized hodograph transformation. In the following two sections we will give applications of this transformation to both elliptic and hyperbolic problems which arise in the study of supersonic flow past a pointed body. We believe that such transformation should be also useful in solving other free boundary value problems.

2. Application to an elliptic problem. Consider a uniform supersonic flow passing a non-symmetric cone. We assume that the cone has straight generating lines and the cross section of a plane perpendicular to the axis of the cone is a slight perturbation to a circle. If the vertex angle of the cone is less than a critical value then the flow will produce a conical shock front attached on the tip of the cone. Assume that the incoming flow is uniform with velocity $\left(q_{\infty}, 0\right)$ and density $\rho_{\infty}$. The flow behind the shock front can be described by the following potential flow equation for the velocity potential $\phi$ :

$$
\sum_{i=1}^{3} \partial_{x_{i}}\left(\phi_{x_{i}} H\right)=0
$$

under the assumption that the flow is isentropic and irrotational. Such an assumption is reasonable for weak shock.

Let the surface of the conical body be given by $m\left(x_{1}, x_{2}, x_{3}\right)=0$, and the shock attached to the tip of the body be given by $\mu\left(x_{1}, x_{2}, x_{3}\right)=0$. Then on the surface $m\left(x_{1}, x_{2}, x_{3}\right)=0$, the boundary condition is

$$
m_{x_{1}} \phi_{x_{1}}+m_{x_{2}} \phi_{x_{2}}+m_{x_{3}} \phi_{x_{3}}=0
$$

On the shock front, the potential $\phi$ is continuous across the shock front

$$
\phi=\phi_{-}\left(=q_{\infty} x_{3}\right)
$$

because the velocity of the coming flow is constant. Moreover, the derivatives of $\phi$ satisfy Rankine-Hugoniot condition

$$
\left(\mu_{x_{1}} \phi_{x_{1}}+\mu_{x_{2}} \phi_{x_{2}}+\mu_{x_{3}} \phi_{x_{3}}\right) H=\mu_{x_{3}} q_{\infty} \rho_{\infty}
$$

Since the problem is invariant under the dilation $x_{i} \rightarrow \alpha x_{i},(i=1,2,3)$ with $\alpha>0$, we can introduce the homogenenus coordinates $\xi=x_{1} / x_{3}, \eta=x_{2} / x_{3}$ and set 
$\phi\left(x_{1}, x_{2}, x_{3}\right)=x_{3} \psi\left(\frac{x_{1}}{x_{3}}, \frac{x_{2}}{x_{3}}\right)$ to obtain an equation for $\psi$. Moreover, by using the polar coordinates $r=\left(\xi^{2}+\eta^{2}\right)^{\frac{1}{2}}, \theta=\arctan (\eta / \xi)$, the equation is reduced to

$$
\begin{gathered}
a^{2}\left(\left(1+r^{2}\right) \psi_{r r}+\frac{1}{r} \psi_{r}+\frac{1}{r^{2}} \psi_{\theta \theta}\right) \\
-\left(\left(1+r^{2}\right) \psi_{r}-r \psi\right)^{2} \psi_{r r}-\frac{2}{r^{2}}\left(\left(1+r^{2}\right) \psi_{r}-r \psi\right) \psi_{\theta} \psi_{r \theta} \\
-\frac{1}{r^{4}} \psi_{\theta}^{2} \psi_{\theta \theta}-\frac{1}{r^{3}} \psi_{r} \psi_{\theta}^{2}+\frac{2}{r^{3}}\left(\psi_{r}\left(1+r^{2}\right)-r \psi\right) \psi_{\theta}^{2}=0 .
\end{gathered}
$$

Assume that the surface of the conical body is $r=b(\theta)$ which is close to a constant $b_{0}$, then the boundary conditions for the physical problem is

$$
\left(1+b^{2}\right) \psi_{r}-\frac{b^{\prime}}{b^{2}} \psi_{\theta}-b \psi=0
$$

on this surface and

$$
\begin{gathered}
\psi=\psi_{0}\left(=q_{\infty}\right), \\
\left(\left(1+s^{2}\right) \psi_{r}-\frac{s^{\prime}}{s^{2}} \psi_{\theta}-s \psi_{0}\right) H+s \psi_{0} \rho_{\infty}=0
\end{gathered}
$$

on the shock front. The shock front $r=s(\theta)$ is a free boundary, which should be determined together with the solution $\psi$. The nonlinear problem (2.5)-(2.8) will be called $(N L)$ ( or $(N L)\{b(\theta)\})$.

We emphasize that the boundary $r=s(\theta)$ is unknown, which should be determined together with the potential $\psi$. To overcome the difficulty caused by the free boundary, we introduce a partial hodograph transformation $T:(r, \theta) \mapsto(p, \sigma)$ :

$$
\left\{\begin{array}{l}
\sigma=\theta \\
p=\psi(r, \theta)
\end{array}\right.
$$

Its inverse transform is $T^{-1}$ :

$$
\left\{\begin{array}{l}
\theta=\sigma \\
r=u(p, \sigma) .
\end{array}\right.
$$

In the new coordinates, the shock front becomes a fixed boundary $p=\psi_{0}$ because the potential $\psi$ outside the shock equals a constant $\psi_{0}$. Meanwhile, the interior domain becomes $\left\{(p, \sigma): p<\psi_{0}\right\}$ and $u(p, \sigma)$ becomes the new unknown function which satisfies $u\left(\psi_{0}, \sigma\right)=s(\theta)$ on the shock front.

The function $u(p, \sigma)$ satisfies a second order differential equation :

$$
\begin{aligned}
& a^{2}\left(-\frac{u_{p p}}{u_{p}^{3}}\left(1+u^{2}\right)+\frac{1}{u u_{p}}+\frac{2 u_{\sigma}}{u^{2} u_{p}^{2}} u_{p \sigma}-\frac{1}{u^{2} u_{p}} u_{\sigma \sigma}-\frac{u_{\sigma}^{2}}{u^{2} u_{p}^{3}} u_{\sigma \sigma}\right) \\
& +\left(\frac{1+u^{2}}{u_{p}}-u p\right)^{2} \frac{u_{p p}}{u_{p}^{3}}+\frac{2}{u^{2}}\left(\frac{1+u^{2}}{u_{p}}-u p\right) \frac{u_{\sigma}}{u_{p}}\left(\frac{u_{\sigma}}{u_{p}^{3}} u_{p p}-\frac{u_{p \sigma}}{u_{p}^{2}}\right)
\end{aligned}
$$




$$
-\frac{1}{u^{4}} \frac{u_{\sigma}^{2}}{u_{p}^{2}}\left(-\frac{u_{\sigma \sigma}}{u_{p}}+\frac{2 u_{\sigma}}{u_{p}^{2}} u_{p \sigma}-\frac{u_{\sigma}^{2}}{u_{p}^{3}} u_{p p}\right)-\frac{u_{\sigma}^{2}}{u^{3} u_{p}^{3}}+\frac{2}{u^{3}}\left(\frac{1+u^{2}}{u_{p}}-u p\right) \frac{u_{\sigma}^{2}}{u_{p}^{2}}=0 .
$$

The boundary conditions become

$$
\begin{gathered}
u=b(\sigma) \\
\left(1+b^{2}(\sigma)\right)+\frac{b^{\prime}(\sigma)}{b^{2}(\sigma)} u_{\sigma}-b(\sigma) p u_{p}=0 .
\end{gathered}
$$

Besides, on the shock front $p=\psi_{0}$

$$
\left(\left(1+u^{2}\right)+\frac{u_{\sigma}^{2}}{u^{2}}-u p u_{p}\right) H+u p u_{p} \rho_{\infty}=0 .
$$

The problem (2.11)-(2.14) will be called $(N L)^{*}$ which is equivalent to $(N L)$.

If $b(\theta)=b_{0}$, the problem is with a regular right cone and its solution is known, see [2]. We will call this known solution as the background solution, and denote the corresponding $\psi=\psi_{B}(r)$. We will look for the solution of $(N L)$ near the background solution $\psi_{B}(r)$.

As we mentioned in the section 1 , we use the method of domain decomposition to avoid the situation that the boundary $r=b(\theta)$ becomes a new free boundary. Introduce two constants $r_{1}$ and $r_{2}$, satisfying

$$
b_{0}<r_{2}<r_{1}, \quad \psi_{20}<\psi_{10}<\psi_{0}
$$

where $\psi_{10}=\psi_{B}\left(r_{1}\right)$ and $\psi_{20}=\psi_{B}\left(r_{2}\right)$. Then we consider two boundary value problems in the interior ring $\Omega_{a}: b(\theta)<r<r_{1}, 0 \leq \theta \leq 2 \pi$ and exterior ring $\Omega_{b}: \psi_{20}<p<\psi_{0}, 0 \leq \theta \leq 2 \pi$ respectively.

$$
\begin{aligned}
& (N L)^{(i)}:\left\{\begin{array}{l}
\text { equation }(2.5) \text { in } \Omega_{a}, \\
\text { boundarycondition }(2.6) \text { on } r=b(\theta), \\
\psi=d(\theta) \text { on } r=r_{1} ;
\end{array}\right. \\
& (N L)^{(e)}:\left\{\begin{array}{l}
\text { equation }(2.11) \text { in } \Omega_{b}, \\
\text { boundarycondition }(2.14) \text { on } p=\psi_{0}, \\
\psi=q(\sigma) \text { on } p=\psi_{20} .
\end{array}\right.
\end{aligned}
$$

We will see that the solvability and estimates for the solutions of $(N L)^{(i)}$ and $(N L)^{(e)}$ will lead to the solution of problem $(N L)$ ( and $(N L)^{*}$ ).

To emphasize our main idea, we assume $\left|r_{1}-b_{0}\right|,\left|\psi_{0}-\psi_{20}\right|$ be so small that the comparison principle applies. Otherwise we will have to decompose the domain to a set of sufficient small overlapped rings and introduce a corresponding set of auxiliary problems to solve $(N L)$. The details can be found in [3].

The problem $(N L)^{(i)}$ can be linearized at the background solution $\psi=$ $\psi_{B}(r), b(\theta)=b_{0}$ and $d(\theta)=\psi_{10}$. The linearized problem for the perturbation $\dot{\psi}$ is

$$
\mathbf{L}^{(i)} \dot{\psi}=A_{11} \dot{\psi}_{r r}+A_{22} \dot{\psi}_{\theta \theta}+B_{1} \dot{\psi}_{r}+C \dot{\psi}=f
$$

with boundary conditions

$$
\left(1+b_{0}^{2}\right) \dot{\psi}_{r}-b_{0} \dot{\psi}=g \quad \text { on } \quad r=b_{0},
$$




$$
\dot{\psi}=h \quad \text { on } \quad r=r_{1} .
$$

The equation (2.18) is elliptic and there exists a unique solution provided $\left|r_{1}-b_{0}\right|$ being small. Moreover, the estimates of Schauder type for the solution are available, i.e.,

LEMMA 2.1. There is $\delta_{1}>0$, such that the solution of $\mathbf{L}^{(i)}$ uniquely exists, and

$$
\begin{aligned}
& \|\dot{\psi}\|_{C^{2+\alpha}\left[b_{0}, r_{1} ; 0,2 \pi\right]} \leq C_{1}\left(\|f\|_{C^{\alpha}\left[b_{0}, r_{1} ; 0,2 \pi\right]}+\|g\|_{C^{1+\alpha}(0,2 \pi)}+\|h\|_{C^{2+\alpha}(0,2 \pi)}\right) \\
& \|\dot{\psi}\|_{C^{2+\alpha}\left[b_{0}, r_{1}-\frac{\delta}{10} ; 0,2 \pi\right]} \leq C_{2}\left(\|f\|_{C^{\alpha}\left[b_{0}, r_{1} ; 0,2 \pi\right]}+\|g\|_{C^{1+\alpha}(0,2 \pi)}+\|h\|_{C^{0}(0,2 \pi)}\right)
\end{aligned}
$$

provided $\left|r_{1}-b_{0}\right|<\delta_{1}$.

Then by the implicit theorem in Banach space, we obtain the existence of solution for $(N L)^{(i)}$. In addition, we have the following comparison principle.

Lemma 2.2. Denote by $(N L)^{(i)}\{b(\theta), d(\theta)\}$ the solution of (2.17) and let $\psi_{j}(r, \theta)=(N L)^{(i)}\left\{b(\theta), d_{j}(\theta)\right\}$. Then $d_{2} \geq d_{1}$ implies $\psi_{2} \geq \psi_{1}$.

By the same argument we can establish the existence and estimates of the solution to the problem $(N L)^{(e)}$ in the exterior ring, and also the comparison principle provided $\left|\psi_{0}-\psi_{20}\right|$ being small.

Similarly as in section 1 , we can establish two sequences $\left\{\psi^{(n)}\right\}$ and $\left\{u^{(n)}\right\}$ beginning from the background solution $\psi_{B}$. The iteration process is as follows.

$$
\begin{aligned}
& \psi^{(1)}(r, \theta)=N L^{(i)}\left\{b(\theta), \psi_{B}\left(r_{1}\right)\right\} \\
& u^{(n)}(p, \sigma)=N L^{(e)}\left\{\left(\operatorname{Inv}\left(\psi^{(n)}\right)\right)\left(\psi_{20}, \sigma\right)\right\} \quad \text { for } \quad n \geq 1 \\
& \psi^{(n+1)}(r, \theta)=N L^{(i)}\left\{b(\theta),\left(\operatorname{Inv}\left(u^{(n)}\right)\right)\left(r_{1}, \theta\right)\right\} \quad \text { for } n \geq 1
\end{aligned}
$$

Using Lemmas 2.1 and 2.2, we can prove the following facts inductively:

1. each $\psi^{(n)}(r, \theta)$ and $u^{(n)}(p, \sigma)$ are bounded from above and below;

2. each $\psi^{(n)}\left(r_{1}, \theta\right)$ is in a $C^{2+\alpha}$ neighborhood of $\psi_{B}\left(r_{1}\right)$, while each $u^{(n)}\left(\psi_{20}, \sigma\right)$ is in a $C^{2+\alpha}$ neighborhood of $u_{B}\left(\psi_{20}\right)$;

3. $\left\{\psi^{(n)}\right\}$ is monotonically decreasing with respect to $u$, while $\left\{u^{(n)}\right\}$ is monotonically increasing.

Combining these facts we can easily arrive at the conclusion that the sequences $\left\{\psi^{(n)}\right\}$ and $\left\{u^{(n)}\right\}$ are convergent. The limit functions $\psi$ and $u$ are inverse functions of each other in the overlapped domain $r_{1} \leq r \leq r_{2}\left(\psi_{10} \geq \psi \geq \psi_{20}\right)$. Therefore the original problem $(N L)$ is solved.

3. Application to a hyperbolic problem. Consider the supersonic flow past a more general pointed solid body with a non-symmetrically curved conic surface. Since the curved cone does not have straight generating lines, the homogeneous coordinates does not reduce the problem into a two-dimensional one. We introduce instead coordinates

$$
r=\left(\left(\frac{x_{1}}{x_{3}}\right)^{2}+\left(\frac{x_{2}}{x_{3}}\right)^{2}\right)^{\frac{1}{2}}, \quad \theta=\arctan \left(\frac{x_{2}}{x_{1}}\right), \quad z=x_{3} .
$$

Correspondingly, the surface of the body and the shock front are denoted by $r=b(z, \theta)$ and $r=s(z, \theta)$ respectively. Consider the equation in the region $b(z, \theta)<r<s(z, \theta)$ and employ the transformation $t=\log z$ to blow up the vertex, we can change the problem into the form 


$$
\begin{gathered}
L \chi \equiv a_{00}\left(\chi_{t}+\chi_{t t}\right)+a_{11} \chi_{r r}+a_{22} \chi_{\theta \theta}+2 a_{01} \chi_{t r}+2 a_{02} \chi_{t \theta}+2 a_{12} \chi_{r \theta} \\
+\left(a_{1}+2 a_{01}\right) \chi_{r}+\left(a_{2}+2 a_{02}\right) \chi_{\theta}=0 \\
E \chi \equiv\left(b+b_{t}\right)\left(\chi+\chi_{t}\right)+\frac{b_{\theta}}{r^{2}} \chi_{\theta}-\left(1+r\left(b+b_{t}\right)\right) \chi_{r}=0 \\
G(\chi) \equiv\left[\chi_{r}^{2}+r^{-2} \chi_{\theta}^{2}+\left(\chi_{t}-r \chi_{r}\right)^{2}\right] H+\left(\chi_{t}-r \chi_{r}\right) q_{\infty}\left(H-\rho_{\infty}\right)=0
\end{gathered}
$$

where $\chi=\phi / x_{3}$, and where the fact $\phi=q_{\infty}$ on the shock front has been employed.

The equation (3.1) is hyperbolic with respect to $t$ and the method of domain decomposition does not work here. Therefore, we use the generalized hodograph transformation like (1.11), i.e.,

$$
y_{0}=t, \quad y_{1}=\theta, \quad y_{2}=\frac{r-b}{r-b+q_{\infty}-\chi} .
$$

Under the transformation, it is obvious that the boundary $r=b(z, \theta)$ and $r=s(z, \theta)$ are transformed to $y_{2}=0$ and $y_{2}=1$ respectively. Meanwhile, we choose

$$
\omega\left(y_{0}, y_{1}, y_{2}\right)=d\left(y_{0}, y_{1}, r\left(y_{0}, y_{1}, y_{2}\right)\right)
$$

as new unknown function, where $d(t, \theta, r)=r-b(t, \theta)+u_{\infty}-\chi(t, \theta, r), r\left(y_{0}, y_{1}, y_{2}\right)$ is the inverse function determined by the last equation of (3.4). We notice that the choice of the independent variables and the new unknown function is equivalent to the combination of the following transformations:

1) Transformation of unknown function

$$
H(t, \theta, r)=\frac{r-b}{r-b+q_{\infty}-\chi(t, r, \theta)}
$$

2) Partial hodograph transformation

$$
y_{0}=t, y_{1}=\theta, y_{2}=H(t, r, \theta)
$$

3) Transformation of unknown function

$$
\omega\left(y_{0}, y_{1}, y_{2}\right)=\left(r-b\left(y_{0}, y_{1}\right)\right) / y_{2} \text {. }
$$

Therefore, denoting $(t, \theta, r)$ by $\left.\left(\xi_{0}, \xi_{1}, \xi_{2}\right), \frac{\partial\left(\xi_{0}, \xi_{1}, \xi_{2}\right)}{\partial\left(y_{0}, y_{1}, y_{2}\right)}\right)$ by $J$, and $\frac{\partial\left(\omega_{y_{0}}, \omega_{y_{1}}, \omega_{y_{2}}\right)}{\partial\left(d_{\xi_{0}}, d_{\xi_{1}}, d_{\xi_{2}}\right)}$ by $\tilde{J}, \chi_{r}(r-b)+q_{\infty}-\chi$ by $d_{1}$, we have

$$
\tilde{J}=\left(\begin{array}{ccc}
\frac{d}{d_{1}} & 0 & \frac{\left(d b_{t}+d_{t}(r-b)\right) d}{d_{1}^{2}} \\
0 & \frac{d}{d_{1}} & \frac{\left(d b_{\theta}+d_{\theta}(r-b)\right) d}{d_{1}^{2}} \\
0 & 0 & \frac{d^{3}}{d_{1}^{2}}
\end{array}\right)=\frac{d}{d_{1}} J^{T}
$$


The equation (3.1) is then reduced to a quasilinear equation for $\omega$

$$
\mathbf{P} \omega \equiv \sum_{i, j=0,1,2} \alpha_{i j}(\omega, \nabla \omega) \omega_{y_{i} y_{j}}+R(\omega, \nabla \omega)=0
$$

in the domain $(-\infty,-T) \times[0,2 \pi] \times(0,1)$. Here the relation $\cdot\left(\alpha_{i j}\right)$ and $\left(a_{i j}\right)$ is

$$
\left(a_{i j}\right)=J^{T}\left(\alpha_{i j}\right) J
$$

Therefore, it is easy to see that the generalized hodograph transformation (3.4) does not change the hyperbolicity of the equation as well as the time-like direction.

The corresponding boundary condition on the surface of the body is

$$
\mathbf{Q}_{\mathbf{b}} \omega \equiv \tilde{M} \nabla_{y} \omega+R_{b}(\omega)=0 \quad \text { on } \quad y_{2}=0,
$$

where $\tilde{M}=M\left(J^{T}\right)^{-1}, M$ is the vector formed by the coefficients of $\nabla_{\xi} \chi$ in (3.2). On the other hand, the boundary condition on the shock front is

$$
\mathbf{Q}_{\mathbf{s}} \omega \equiv \tilde{G}\left(\omega, \omega_{y_{0}}, \omega_{y_{1}}, \omega_{y_{2}}\right)=0 \quad \text { on } \quad y_{2}=1
$$

We notice here that the relation

$$
\nabla_{q} \tilde{G}=\nabla_{p} G \tilde{J}^{-1}
$$

holds, where $q, p$ stand for the arguments $\nabla_{y} \omega, \nabla_{\xi} \chi$ in the nonlinear functions $\tilde{G}$ and $G_{1}$.

The problem (3.9)-(3.11) is a boundary value problem of a quasilinear hyperbolic equation with fixed boundary. It can be solved by using Newton's iterative scheme combining with the energy method in a suitably weighted Sobolev space. We will use the result in the previous section to obtain the the approximate solution used as the first term in the iteration process.

For a given pointed body, we make its tangent cone. This tangential cone is generally non-symmetric. The solution of the same upstream supersonic flow passing the tangent cone can be obtained by the method in Section 2 . When the given pointed body and its tangent cone are osculatory at the tip up to sufficiently high order, the solution $\tilde{\omega}$ corresponding to the tangent cone can be taken as the first approximation in the linear iteration of the nonlinear problem. By inductively solving the following linearized problem

$$
\begin{aligned}
& P\left(\tilde{\omega}+\dot{\omega}_{k}\right) \dot{\omega}_{k+1} \equiv \sum_{i, j=0}^{2} \alpha_{i j}\left(\tilde{\omega}+\dot{\omega}_{k}\right) \partial_{y_{i}} \partial_{y_{j}} \dot{\omega}_{k+1} \\
& \quad+\sum_{j=0}^{2} \alpha_{j}\left(\tilde{\omega}+\dot{\omega}_{k}\right) \partial_{y_{j}} \dot{\omega}_{k+1}+\alpha\left(\tilde{\omega}+\dot{\omega}_{k}\right) \dot{\omega}_{k+1}=f_{k} \text { in } 0<y_{2}<1
\end{aligned}
$$

$$
\begin{aligned}
& Q_{b}\left(\tilde{\omega}+\dot{\omega}_{k}\right) \dot{\omega}_{k+1} \equiv \sum_{j=0}^{2} \beta_{1 j}^{(k)} \partial_{y_{j}} \dot{\omega}_{k+1}+\beta_{0}^{(k)} \dot{\omega}_{k+1}=g_{1 k} \text { on } y_{2}=0 \\
& Q_{s}\left(\tilde{\omega}+\dot{\omega}_{k}\right) \dot{\omega}_{k+1} \equiv \sum_{j=0}^{2} \gamma_{1 j}^{(k)} \partial_{y_{j}} \dot{\omega}_{k+1}+\gamma_{0}^{(k)} \dot{\omega}_{k+1}=g_{2 k} \text { on } y_{2}=1
\end{aligned}
$$


with $\dot{\omega}_{0}=0$, we can construct a sequence $\left\{\dot{\omega}_{k}\right\}$, which is bounded in higher weighted Sobolev norm and is convergent in lower weighted Sobolev norm by the energy estimate of linearized problem. The limit of $\tilde{\omega}+\dot{\omega}_{k}$ is the solution of (3.11)-(3.13).

We remark that when the above-mentioned high order osculation is not satisfied, an additional treatment is necessary. This treatment involves introducing a finite power expansion and solving a set of related boundary value problem in order to modify the first approximation. The details can be found in [4] and [7].

The method of generalized hodograph transformation can also be applied to other free boundary problems. For instance, to Stefen problem of parabolic equation in multidimensional space (see $[8,12]$ ), to the problem of determination of a shock front produced by a supersonic flow inside a duct, see [5].

Acknowledgment. This work is partially supported by NSF of China, Key Grant of NMST of China, Doctoral Foundation of NEM and WVU Foundation. The first author also would like to thank City University of Hong Kong and IMS of CUHK where he worked on this topic in his visiting period.

\section{REFERENCES}

[1] Blokhin, A. AND BiRkin, A., Global solvability of the problem of supersonic flow around a cone, Mat. Model, 4 (1996), pp. 89-104.

[2] Courant, R. And Friedrichs, K.O., Supersonic Flow and Shock Waves, Interscience Publishers Inc., New York, 1948.

[3] Chen, S.X., Non-symmetric conical supersonic flow, International Series of Numer. Math., Birkhäuser Verlag, Basel/Switzerland, 129 (1999), pp. 149-158.

[4] Chen, S.X., Local existence if solution to supersonic flow past a pointed body, in preparation.

[5] ChEn, S.X. AND XIN, Z.P., Supersonic flow in two-dimensional duct, in preparation.

[6] Chen, S.X. AND LI, D.N., Supersonic flow past a symmetrically curved cone, Indiana Univ. Math. Jour., 49 (2000), pp.1411-1435.

[7] Chen, S.X., Geng Z. W. AND Li, D.N., Perturbation and stability of conic shock waves, to appear.

[8] LI, D.N., A one-phase hyperbolic Stefan problem in multi-dimensional space, Trans. Amer. Math. Soc., 318 (1990), pp. 401-415.

[9] LI, D.N., Rarefaction and shock waves for multi-dimensional hyperbolic conservation laws, Comm. in PDEs, 16 (1991), pp. 425-450.

[10] MAJDA, A., One perspective on open problems in multi-dimensional conservation laws, Multidimensional Hyperbolic Problems and Computation, Springer Verlag, IMA 29 (1990), pp. $217-237$.

[11] Majda A. AND Thomann, E., Multi-dimensional shock fronts for second order wave equations, Comm. P.D.E., 12 (1987), pp. 777-828.

[12] MeIrmanov, A.M., On a classical solution of the multidimensional Stefan problem for quasilinear parabolic equations, Math. Sb., 112 (1980), pp. 170-192.

[13] MNIF, M. AND SFAX, I.P.E., Probleme de Riemann pour une loi conservation scalaire hyperbolique d'order deux, Comm. in PDEs, 22 (1997), pp. 1589-1627. 